Cahiers de civilisation médiévale

\title{
Collectif, Personal Names and Naming Practices in Medieval Scotland
}

\section{Arnaud Lestremau}

\section{(2) OpenEdition}

1 Journals

Édition électronique

URL : https://journals.openedition.org/ccm/7548

DOI : $10.4000 / \mathrm{ccm} .7548$

ISSN : 2119-1026

Éditeur

Centre d'études supérieures de civilisation médiévale/Université de Poitiers

\section{Édition imprimée}

Date de publication : 1 juin 2021

Pagination : 183-186

ISBN : 978-2-490783-09-0

ISSN : 0007-9731

\section{Référence électronique}

Arnaud Lestremau, "Collectif, Personal Names and Naming Practices in Medieval Scotland ", Cahiers de civilisation médiévale [En ligne], 254 | 2021, mis en ligne le 01 juin 2021, consulté le 30 novembre 2022 URL : http://journals.openedition.org/ccm/7548; DOI : https://doi.org/10.4000/ccm.7548

\section{c) (†) $\ominus$}

Creative Commons - Attribution - Pas d'Utilisation Commerciale - Pas de Modification 4.0 International - CC BY-NC-ND 4.0

https://creativecommons.org/licenses/by-nc-nd/4.0/ 
Personal Names and Naming Practices in Medieval Scotland, Matthew Hammond (dir.), Woodbridge/Suffolk, The Boydell Press (Studies in Celtic History, 39), 2019.

L'ouvrage est le compte rendu tardif et partiel d'une journée d'études qui a eu lieu au Centre for Scottish and Celtic Studies (Université de Glasgow), le 15 mars 2014, dans le cadre du programme AHRC " Paradox of Medieval Scotland ». Il illustre les possibilités offertes par le fabuleux outil mis en ligne par les équipes de Dauvit Broun (Glasgow), avec la participation de King's College (Londres), à savoir la base prosopographique People of Medieval Scotland, 1093-1371 (https://www.poms.ac.uk/). Le livre n'est pas une transcription exacte des discussions qui ont eu lieu en 2014, puisque plusieurs conférences n'ont pu être publiées et ont été remplacées par d'autres papiers, sélectionnés par l'éditeur. C'est le cas des contributions de Matthew Hammond (chap. 4), David Sellar (chap. 5), Valeria Di Clemente (chap. 6) et Tom Tupie (chap. 9). La bibliographie générale de 23 p. est un précieux outil, qui inclut quelques éléments importants de la bibliographie continentale (Monique Bourin et Pascal Chareille, notamment), mais elle penche de façon excessive vers la littérature anglo-gaélique et semble ignorer les travaux importants menés sur des thèmes similaires dans d'autres pays, par ex. par Michael Mitterauer. On peut regretter que l'éditeur n'y ait pas distingué sources primaires et sources secondaires, ni que, dans l'index de $21 \mathrm{p}$. exclusivement consacré aux noms propres, il n'ait pas séparé anthroponymes et toponymes. Toutefois, malgré ces menues critiques, l'ouvrage est très bien édité et compte très peu de coquilles.

L'introduction (M. Hammond, chap. 1) est un très beau travail de synthèse sur le lien entre noms et identités. L'a. y brasse les principales sources 
d'inspiration pour ceux qui choisissent un nom (piété, parenté, seigneurie, etc.), précise les langues d'origine des noms présentes en Écosse et replace le cadre de la « révolution anthroponymique » des $\mathrm{XI}^{\mathrm{e}}-\mathrm{XIII}^{\mathrm{e}} \mathrm{S}$, montrant divers cas illustrant le passage d'un système uninominal à un système " moderne » à deux noms. Il y présente aussi l'outil People of Medieval Scotland (21 000 acteurs, 600 documents) et en explique les déséquilibres (sous-représentation des femmes, des classes populaires, du nord de l'Écosse). Avant de proposer un résumé rapide des contributions, il présente enfin une bibliographie critique des outils susceptibles d'être utilisés pour parler des noms en Écosse médiévale et contemporaine, en Irlande, en Angleterre anglo-saxonne et en Angleterre contemporaine. À n'en pas douter, il s'agit d'un outil commode et efficace pour qui désirerait s'initier au sujet.

Le chap. 2 (Nicholas Evans) étudie de manière diachronique la place des noms dans les chroniques gaéliques, en établissant une comparaison entre l'Irlande (Annals of Ulster, pour les années 661-760 et 901-1000) et l'Écosse (Chronicle of the Kings of Alba, pour les $\mathrm{IX}^{\mathrm{e}}-\mathrm{X}^{\mathrm{e}} \mathrm{s}$.). Après avoir présenté ses sources et leurs biais, il présente sa population : 908 noms pour 785 individus (en 661-760), 49 noms (pour la période centrale) et 724 noms pour 531 individus (en 901-1000). Les biais sont classiques : peu de femmes (1,72\% des noms), sous-représentation des catégories populaires et du nord de l'Écosse. La comparaison diachronique laisse apparaître un changement en Irlande, avec le remplacement progressif des noms simples, éventuellement accompagnés d'un toponyme ou d'un titre, par un système à deux noms recourant de façon croissante à l'usage de patronymiques et des termes Ua ou Nepos, avec les sens variables de « petit-fils » ou « descendant mâle ». Par contraste, en Écosse, les patronymiques sont plus nombreux d'entrée et Ua/Nepos est peu utilisé. Dans le même temps, l'a. observe la réduction importante du stock de noms, qui tend à se christianiser (usage de noms théophores ou hagiophores) et à se concentrer dans plusieurs parentèles sur un nombre réduit de noms marqueurs. En Écosse, particulièrement, on observe aussi l'influence croissante des vikings (et du stock norrois) sur le système anthroponymique.

Roibeard O Maolalaigh (chap. 3) dresse une étude complète de l'onomastique gaélique en utilisant notamment l'outil People of Medieval Scotland. Au total, il a utilisé 6000 documents, a identifié 15200 individus dont 1500 anonymes, portant environ 1300 noms différents. Outre les habituelles limites (sous-représentation des femmes, des milieux populaires et des habitants du nord et de l'ouest de l'Écosse), il constate la grande dispersion du stock (65\% des noms ne sont portés qu'une fois) et la grande condensation sur quelques noms très populaires. Il établit pour le démontrer plusieurs listes des noms les plus portés et en identifie la langue d'origine. L'a. a parfaitement conscience des difficultés de cette démarche (noms traduits d'une langue à l'autre, noms pouvant avoir plusieurs origines, etc.) et il les explique avec clarté et lucidité, tout en proposant d'habiles analyses pour éviter les erreurs. $15 \%$ d'entre eux sont sans doute gaéliques, soit 255 noms différents, portés par 1237 individus dans 6751 occurrences documentaires. Les noms les plus usuels sont les théophores reposant sur des formes composées du gaélique Mael ou Gilla, « serviteur, dévoué », suivi du nom d'un saint ( $34 \%$ de la population). L'essentiel de l'article identifie les occurrences et analyse le sens et les formes des noms, notamment en distinguant celles qui ont pour origine un adjectif, un nom, etc. Il ne s'agit donc pas d'un article qu'on peut lire avec plaisir, mais d'un outil absolument incontournable et qui fera date.

Dans son article (chap. 4) sur les surnoms emblématiques en Écosse, M. Hammond apporte un éclairage complémentaire à celui du chap. 2. Il s'agit d'une analyse serrée sur trois surnoms « classiques » dans le monde gaélique : $M a c, U a$ et $M a c$ Meic, dont les sens ont cependant connu d'importantes vicissitudes entre $\mathrm{XI}^{\mathrm{e}}$ et XIII ${ }^{\mathrm{e}}$ s. Pour l'a., tous ces surnoms ont d'abord été utilisés comme des phrases relationnelles, descriptives, renvoyant donc à une réalité généalogique, avant de se figer comme surnoms héréditaires, sinon comme revendication politique ou comme marque de légitimité pour diriger un clan. Apparu pour désigner le «petit-fils » au XI ${ }^{\mathrm{e}} \mathrm{s}$., $U a$ se fige et signifie rapidement « descendant masculin », ce qui donna lieu à l'apparition au cours des deux siècles suivants de la phrase relationnelle Mac Meic, «fils du fils de », lequel s'est progressivement figé au XIII ${ }^{\mathrm{e}} \mathrm{s}$. Le surnom Mac, « fils de », est apparu à la charnière des $\mathrm{XII}^{\mathrm{e}}$-XIII ${ }^{\mathrm{e}} \mathrm{s}$, d'abord en Irlande comme abréviation de Mac Meic, puis en Écosse, directement et sans étape intermédiaire reposant sur l'abréviation de Mac Meic. Ce surnom, 
à son tour, s'est rapidement fossilisé pour désigner les descendants d'un ancêtre éponyme. Outre l'annexe, reposant sur l'outil People of Medieval Scotland et présentant les cas dans lesquels ces surnoms sont utilisés, l'a. présente au fil de l'article de nombreux exemples circonstanciés pour appuyer son analyse. Cela rend son texte difficile à contredire, mais aussi difficile à lire pour qui ne connaîtrait pas parfaitement les parentèles de l'Écosse du Moyen Âge central.

L'article 5 est une republication d'un article de D. Sellar (dans Regions and Rulers in Ireland, 1100-1650: Essays for Kenneth Nicholls, David EDwARDs [dir.], Dublin, Four Courts [Cork Studies in History \& Culture, 4], 2004). Il s'agit d'un texte extrêmement court visant à établir l'identité entre les formes latine Forflissa, gaélique Forbflaith et norroise Hvarflöd d'un même nom. Ayant procédé à cette identification, l'a. établit une liste des occurrences connues de ce nom dans les sources. En annexe, M. Hammond ajoute les occurrences supplémentaires mises au jour grâce au projet People of Medieval Scotland.

V. Di Clemente (chap. 6) s'intéresse aux noms d'origine germanique présents dans le Ragman Roll, un document de 1296 dans lequel le roi Edward I ${ }^{\text {er }}$ fait inscrire les noms d'Écossais qui lui ont prêté serment de fidélité. Le document compte 1900 occurrences pour un total d'environ 1650 individus. Après avoir décrit les manuscrits préservés et les éditions de référence, l'a. rappelle rapidement par quels biais une influence onomastique germanique a pu modifier le stock écossais (influence des cultes anglo-saxons, alliances réalisées par la famille royale, etc.). L'essentiel de l'article est constitué d'un catalogue des noms, avec identification de leur sens et références aux linguistes qui font autorité sur ce dossier. Les formes des noms sont également décrites : nombreux noms bithématiques, rares noms monothématiques, présence de diminutifs, cas de francisation de noms anglo-saxons ou scandinaves ou de latinisation de noms germaniques. Comme au chap. 3, la lecture de l'article n'est pas en soi intéressante, mais il s'agit d'un outil capital.

Le chap. 7, rédigé par Thomas Owen Clancy et M. Hammond, s'intéresse à l'influence de la littérature populaire sur le choix des noms en Écosse. Après avoir rappelé le cadre de la révolution anthroponymique sous l'angle de l'européanisation des stocks onomastiques et fait un bref rappel sur les trois grands cycles de la littérature médiévale (matière romaine, matière franque et matière de Bretagne), les auteurs précisent quelques difficultés méthodologiques. L'absence de manuscrit préservé d'une œuvre ne signifie pas que cette œuvre était inconnue, puisque sa renommée a pu circuler par le bouche-à-oreille (ou le manuscrit être perdu). La popularité d'un nom issu d'une œuvre littéraire peut ne pas être due à la popularité de cette œuvre, notamment si le premier porteur était lui-même très célèbre : c'est le cas avec le nom Alexandre, d'abord porté en Écosse par un roi très populaire. Parfois des noms se trouvent à la croisée de la tradition littéraire et de la tradition hagiologique : ainsi Hélène peut renvoyer au cycle de Troie ou à la mère de Constantin; il est alors difficile de trancher. De manière générale, le chapitre se résume à un catalogue de noms et à la prosopographie, éventuellement familiale, de leurs porteurs. Si la dimension éphémère de ces effets de mode et la concentration de plusieurs noms de ce type dans quelques groupes familiaux semblent conforter la démarche d'ensemble, le chapitre bute sur la fragilité de la documentation et sur l'absence de discours clairs des acteurs sur les choix onomastiques qu'ils ont pu poser.

Le chap. 8, proposé par John Reuben Davies, tente d'identifier les noms issus de l'Ancien Testament dans l'Écosse médiévale en s'appuyant sur la base People of Medieval Scotland. Les 36 noms hébreux étudiés représentent $10 \%$ du stock pendant la période 10931286, alors qu'ils sont très rares avant 1093 et dans les royaumes voisins. L'a., après avoir montré le caractère invalide $d$ 'une thèse faisant reposer cette relative profusion sur la présence d'importantes communautés juives en Écosse, identifie plusieurs explications : dans un idéal de purification, les Prémontrés ont tendance à adopter des noms issus de l'Ancien Testament; certaines régions d'Écosse, notamment le Lennox et le Strathearn, apprécient également ces noms; enfin, l'influence croisée des Bretons (du continent) et des Anglo-Normands, particulièrement dans un contexte de Croisade, explique sans doute beaucoup de ces noms. L'article est en grande partie constitué d'un catalogue des noms hébreux, de leur sens et de leur origine biblique, avec une prosopographie sommaire de leurs porteurs. Là encore, il s'agit d'un outil précieux.

Les chap. 9 et 10, écrits respectivement par T. Turpie et Rachel Butter, portent sur les noms des saints gaéliques dans l'Écosse de la fin du Moyen Âge et du début de l'ère moderne. T. Turpie se concentre sur 
des listes de migrants écossais en Angleterre (1440), de marins de la Navy sous Jacques IV (1513) et de clercs (1560). En comparant de manière diachronique ces trois listes, il constate une diffusion progressive du nom de saints écossais (Ninian, Mungo, Duthac, etc.), qu'il analyse comme un reflet du nationalisme religieux d'une Écosse alors dominée par l'Angleterre. On peut regretter que l'a. ne fasse pas de remarque sur la différence qui existe entre les trois populations citées dans ces documents : le fait d'être clerc explique peut-être ces différences mieux que le décalage chronologique. De même, il est tout à fait possible que ces noms et ces cultes aient été présents avant et ailleurs, notamment dans les catégories populaires dont les noms sont rarement préservés. Cette absence de prise en compte du horschamp suscite quelques réserves, même si l'analyse de l'apparition de certains noms en corrélation avec la dédicace d'autels à proximité emporte l'adhésion, tant l'approche de l'a. est érudite et sérieuse. C'est avec une démarche similaire que R. Butter s'intéresse aux saints d'Argyll, à l'ouest de l'Écosse. Utilisant deux listes locales de la fin du XVII ${ }^{\mathrm{e}}$ s., l'a. constate le caractère usuel des noms hagiophores construits sur Mael et Gilla. L'étude repose sur une analyse fine de la distribution de ces anthroponymes en comparaison de toponymes Cill. Par ex., trouve-t-on beaucoup de personnes appelées Gilla Adomnain, « serviteur d'Adomnan », à proximité de lieux appelés
Cill Adomnain, " village d'Adomnan »? L'analyse des cas reposant sur les noms Adomnan, Colomba, Sencha et Миппи, permet effectivement d'identifier des cultes régionaux, centrés sur le sanctuaire d'un saint, renforcé par la prolifération d'anthroponymes confortant le rôle de ce saint, par des toponymes hagiophores et promus par des parentèles spécifiques. À la fin de son article, l'a. signale cependant que cette logique disparait progressivement sous l'effet de l'anglicisation des noms et de la Réforme, qui concourent évidemment à détruire le culte des saints gaéliques.

Cet ouvrage, oscillant entre énoncés statistiques, reconstitutions prosopographiques et analyses linguistiques, n'est pas à la portée du lecteur non initié. Les articles thématiques sont rudes et techniques, et manquent parfois de phrases générales permettant de cerner les enjeux de la démonstration. Nul doute, dans le même temps, que les articles construits comme des catalogues de noms constitueront des outils appréciables, incontournables, susceptibles de nourrir les études à venir sur le même thème.

Arnaud LestREMAU Laboratoire de médiévistique occidentale de Paris / Lycée Paul Éluard (Saint-Denis) 